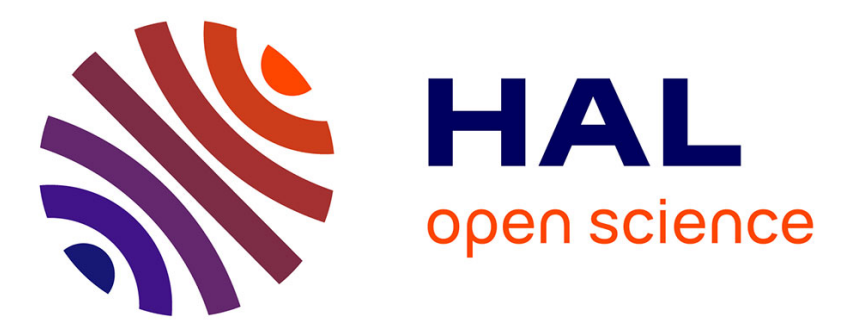

\title{
2D Image-based reconstruction of shape deformation of biological structures using a level-set representation
}

\author{
Ronan Fablet, Sylvain Pujolle, Anatole Chessel, Abdesslam Benzinou,
} Frédéric Cao

\section{- To cite this version:}

Ronan Fablet, Sylvain Pujolle, Anatole Chessel, Abdesslam Benzinou, Frédéric Cao. 2D Imagebased reconstruction of shape deformation of biological structures using a level-set representation. Computer Vision and Image Understanding, 2008, 111 (3), pp.295 - 306. 10.1016/j.cviu.2007.12.005 . hal-02344335

\section{HAL Id: hal-02344335 https://hal.science/hal-02344335}

Submitted on 25 Nov 2019

HAL is a multi-disciplinary open access archive for the deposit and dissemination of scientific research documents, whether they are published or not. The documents may come from teaching and research institutions in France or abroad, or from public or private research centers.
L'archive ouverte pluridisciplinaire HAL, est destinée au dépôt et à la diffusion de documents scientifiques de niveau recherche, publiés ou non, émanant des établissements d'enseignement et de recherche français ou étrangers, des laboratoires publics ou privés. 


\title{
2D Image-based reconstruction of shape deformation of biological structures using a variational level-set representation
}

\author{
R. Fablet ${ }^{1 *}$, S. Pujolle ${ }^{1}$, A. Chessel ${ }^{1}$, A. Benzinou ${ }^{2}$ and F. Cao ${ }^{3}$ \\ Ifremer/LASAA \\ ENIB/RESO \\ IRISA/VISTA \\ BP 70, Tecnopôle Brest-Iroise Tecnopôle Brest-Iroise Campus de Beaulieu \\ 29280 Plouzane, FR 29280 Plouzane, FR 35014 Rennes, FR \\ \{rfablet,achessel\}@ifremer.frｂenzinou@enib.fr $\quad$ fcao@irisa.fr
}

\footnotetext{
${ }^{*}$ Corresponding author (rfablet@ifremer.fr, phone: +33298224388, fax: +33298224653)
} 


\begin{abstract}
This paper copes with the reconstruction of accretionary growth sequence from images of biological structures depicting concentric ring patterns. Accretionary growth shapes are modeled as the level-sets of a potential function. Given an image of biological structure, the reconstruction of the sequence of growth shapes is stated as a variational framework derived from geometric criteria. This variational setting exploits image-based information, in terms of the orientation field of relevant image structures, which leads to an original advection term. The resolution of this variational issue is discussed. Experiments on synthetic and real data are reported to validate the proposed approach.
\end{abstract}

1 Keywords: shape matching, accretionary morphogenesis, level-set, inverse problem

\title{
21 Introduction and problem statement
}

3 A number of biological structures, for instance corals, seashells, fish otoliths ${ }^{1}$, tree trunks or verte4 brae grow according to an accretionary process. In other words, they can be viewed as a succession 5 of three-dimensional concentric layers (with respect to an initial core). The composition of these 6 layers, in terms of crystalline organization and chemical features, vary according to endogenous and 7 exogenous factors [25]. Often, the accretionary process is associated with a periodic, mainly daily

or seasonal, scheme, such that the observation of these biological structures in an observation plane going through the initial core depict concentric ring patterns, also called grwoth marks, as illustrated in Fig.1. These characteristics provide the basis for exploiting these structures as biological archives to define environmental proxies (e.g., for instance to reconstruct temperature and salinity sequences) [8] or to reconstruct individual life traits (e.g., individual age and growth information or migration paths) [16]. To further stress the key importance of these biological structures in marine

\footnotetext{
${ }^{1}$ Fish otoliths are calcified structures present in fish inner ears
} 

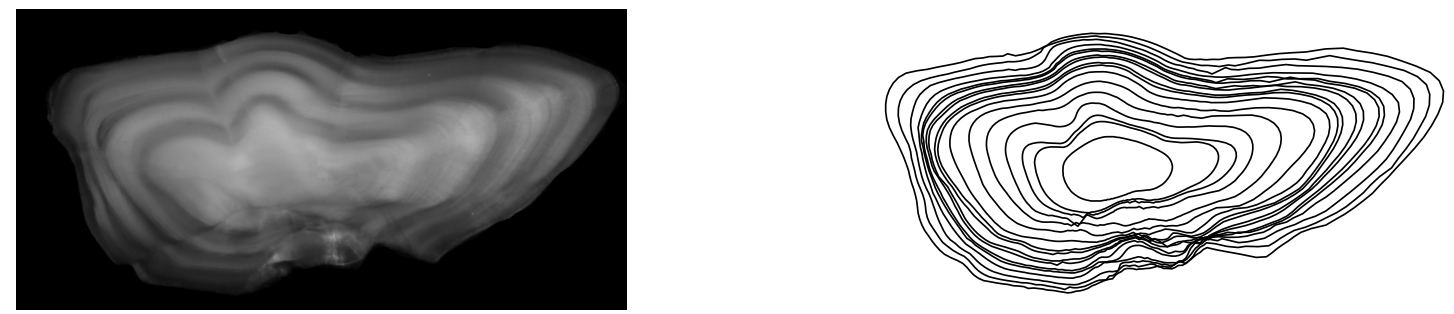

Figure 1: Illustration of the goal of the paper: image of a pollock otolith in a given observation plane containing the growth center (left); reconstructed series of the evolved shapes (right).

1 ecology, it can be pointed out for instance that several millions of fish otoliths are analyzed each 2 year for fisheries management.

3 New computational tools $[15,16]$ are sought to improve the understanding, the modeling and the 4 decoding of these biological archives. Their shape characteristics have been extensively exploited 5 [4]. Though the presence of internal growth rings provide the mean for back-tracking the temporal 6 evolution of the shape from the core to the outline, the actual analysis and reconstruction of this 7 morphogenesis sequence have not been investigated. From an image processing perspective, the 8 reconstruction of the morphogenesis of the considered biological structures within an observation 9 plane $^{2}$ can be viewed as the reconstruction of the shape deformation from the initial circular core to the observed outline as illustrated in Fig.1. Mathematically-sound solutions [6, 30] have been 11 proposed to determine the deformation path between any two shapes. The direct application of these schemes would however first require extracting the internal growth rings. Whereas the detection of the growth center and the detection of the external shape may be automated [5], the automated extraction of internal growth rings is a particularly complex task due to the presence of blind areas and so-called subjective contours $[19,15]$. Image orientations, i.e. the local orientations

\footnotetext{
${ }^{2}$ In the subsequent, the term "observation plane" will only refer to an observation plane containing the initial core of the analyzed biological structure
} 
of image structures [9], however locally convey relevant information on the shape of the growth rings, since they can be viewed as estimations of the local growth directions. Consequently, we state the reconstruction of the accretionary growth sequence as the computation of the deformation shape between the initial core and the outline such that any intermediary shape should be normal to local image orientations.

To this end, we adopt a level-set setting to model the accretionnary growth process. Coupling a priori constraints on shape regularity and image-based information in terms of local shape orientation, the considered issue resorts to a variational level-set interpolation. This paper is organized as follows. Section 2 details the proposed variational framework. Minimization issue are described in Section 3. Experiments and concluding remarks are reported in Sections 4 and 5.

\section{Proposed approach}

\subsection{Level-set setting}

As suggested in the seminal work of Thompson [27], we adopt a level-set setting to represent the accretionary growth process. It comes to introduce a potential function $U$ defined over $\mathcal{R}^{2}$ such that the shape $\Gamma_{t}(U)$ of the considered biological structure within a given observation plane at time $t$ is given by the level set of $U$ :

$$
\Gamma_{t}(U)=\left\{p \in \mathcal{R}^{2} \text { such that } U(p)=f(t)\right\}
$$

where $f$ is a strictly monotonic continuous function. Given $U$, the sequence of level sets $\left\{\Gamma_{t}(U)\right\}_{[0, T]}$ represents the evolution of the shape from time 0 to time $\mathrm{T}$. This representation conforms to the classical assumption that accretionary growth is locally normal to the shape. The growth increment at any point $p$ is indeed inversely proportional to $\nabla U(p)$, which is, by definition, normal in $p$ to 


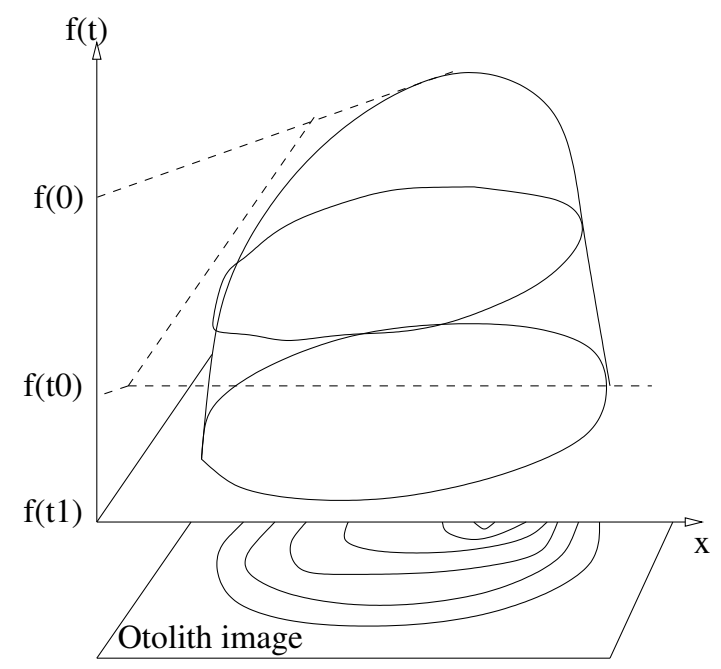

Figure 2: Level-set representation of the accretionary growth process: the evolution of the shape is described by a potential function $U$, such that the growth shape at time $t$ is given by level-line $\Gamma_{t}(U)=\left\{p \in \mathcal{R}^{2}\right.$ such that $\left.U(p)=f(t)\right\}$ with $f$ a continuous and strictly monotonic function.

1 level-set $\Gamma_{U(p)}(U)$.

Let us stress that this level-set representation is generic. Convex as well as non-convex shapes 3 are represented. Besides, secondary growth centers could also be taken into account, though this 4 point is not investigated in this paper.

\section{${ }_{5} \quad 2.2 \quad$ Geometric priors and orientation-based constraints}

6 Given an image of the considered biological structure in a given observation plane, our goal is

7 to reconstruct the potential function $U$ associated with the underlying accretionary growth. Let

8 us stress that the proposed level-set representation provides a relevant framework to solve for the

9 inverse problem, since it intrinsically conveys the key geometric properties of the accretionary 10 growth: concentricity and local parallelism of the successive level-sets. Besides, as shown below, 11 such a representation offers a powerful tool to transform a purely geometric setting to a variational 

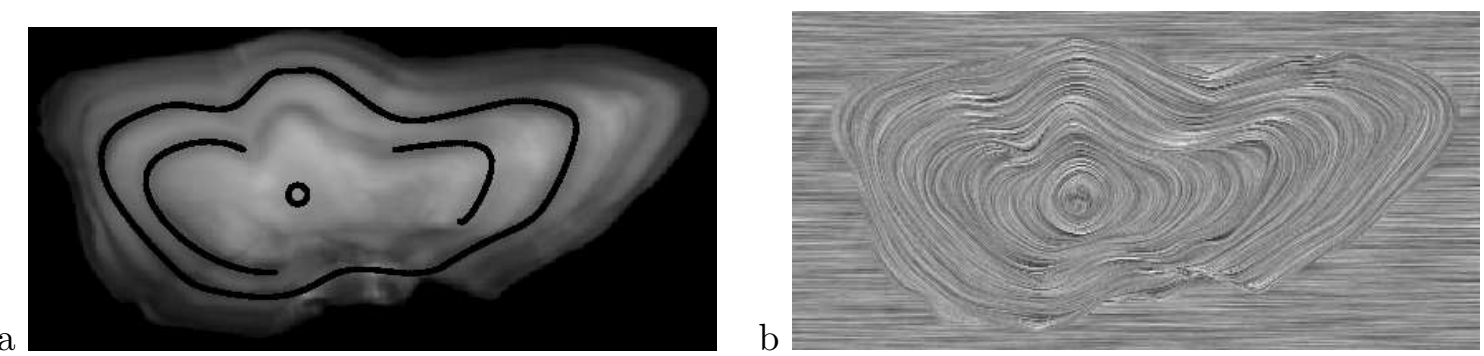

Figure 3: Geometric priors and orientation-based information exploited to constrain the reconstruction of the accretionary morphogenesis: (a) example of boundary conditions given as a point or curve set for which potential values are known (depicted as black curve superimposed to the image of the considered biological structure), (b) example of orientation field computed from the image of the considered biological structure(the orientation field is visualized via its field line using LIC [3]).

minimization issue.

Formally, we assume that we are given with some boundary conditions as illustrated in Fig.2.2.a: at least the shapes at times 0 and $\mathrm{T}$, but additional constraints can be considered such as internal partial or complete growth rings. These conditions are stated as a set of points or curves for which the potential value is known. Let us denote by $\mathcal{B}$ this point set and $G_{\mathcal{B}}$ the associated potential values. Solving for the reconstruction of $U$ is then stated as the computation of its extension from $\mathcal{B}$ to the whole domain $\mathcal{R}^{2}$.

In addition, the image conveys relevant local geometric information in terms of orientations of the ring structures. Formally, we assume that an orientation field normal to local growth orientations is provided as illustrated in Fig.2.2.b. The interpolation of level-set representation $U$ is then be constrained to be locally tangent to this orientation field. A first solution consists in considering orientation field $\omega=\nabla I^{\perp} /|\nabla I|$ with $I$ the image intensity. Obviously, for low gradient values, the computation of the orientation is highly noisy. However, the module of the image gradient can be 

the whole domain $\mathcal{R}^{2}$ is defined as the minimization of an energy criterion $E$ :

$$
\widehat{U}=\arg \min _{U \in \mathcal{U}\left(G_{\mathcal{B}}\right)} E(U)
$$

where $\mathcal{U}\left(G_{\mathcal{B}}\right)$ is the set of the functions from $\mathcal{R}^{2}$ to $\mathcal{R}$ whose restriction to $\mathcal{B}$ is $G_{\mathcal{B}}$. Energy $E(U)$ is split into two terms: a regularization term issued from a geometric shape prior and a data-driven term setting orientation-based constraints,

$$
E(U)=(1-\gamma) E_{R}(U)+\gamma E_{O}(U)
$$

where $\gamma$ is a weighting factor balancing the relative influence of each term.

The chosen regularity criterion resorts to minimizing the perimeter of any growth shape $\Gamma_{t}(U)$. Hence, $E_{R}(U)$ is defined as the sum over all level-sets of the unity function:

$$
E_{R}(U)=\int_{t \in[0, T]} \int_{p \in \Gamma_{t}(U)} 1
$$

The second energy term evaluates how the reconstructed growth shapes conform to local growth orientations $\omega$. Any shape $\Gamma_{t}(U)$ is expected to be normal to orientation field $\omega$. Given that $\Gamma_{t}(U)$ 
is a level-line of $U$, this is equivalent to the orthogonality between growth directions $\nabla U /|\nabla U|$ and orientations $\omega$ along $\Gamma_{t}(U)$. Therefore, summing over all level-lines of $U, E_{O}(U)$ is defined as

$$
E_{O}(U)=\int_{t \in[0, T]} \int_{p \in \Gamma_{t}(U)} \alpha(p) \cdot \rho\left(\left\langle\frac{\nabla U(p)}{|\nabla U(p)|}, \omega(p)\right\rangle\right)
$$

1 where $\rho$ is an error function. In order to account for outliers, a robust function is exploited [20].

2 Compared to a classical quadratic term, it ensures the robustness to local incoherences within 3 orientation field $w$. In the subsequent, $\rho$ is set as $\rho(x)=|x|$.

\section{$4 \quad 2.3 \quad$ Variational framework}

The above definition of the energy criterion is issued from the summation over all level-lines of $U$. The direct minimization of $E(U)$ from this formulation is then infeasible since it first implies to extract all level-lines of $U$. The solution comes from the co-area formula which supplies us with an equivalent computation over $\mathcal{R}^{2}$. More precisely, for any function $\Psi$, the following result applies $[21]:$

$$
\int_{t \in[0, T]} \int_{p \in \Gamma_{t}(U)} \Psi=\int_{p \in \mathcal{R}^{2}}|\nabla U| \Psi
$$

5 Norm $|\nabla U|$, inherited from the co-area transform, can be viewed as a weight which gives more

6 influence to points where the gradient of the level-set representation is high (conversely, where the 7 growth is slow).

Using the formulations of energy terms $E_{R}(U)$ and $E_{R} O(U)$, we resort to the following variational issue:

$$
\widehat{U}=\arg \min _{U \in \mathcal{U}\left(G_{\mathcal{B}}\right)} \int_{p \in \mathcal{R}^{2}}(1-\gamma) \cdot|\nabla U|+\gamma \cdot \alpha \cdot|\langle\nabla U, \omega\rangle|
$$

The reconstruction of the series of growth shapes can then be regarded as a variational inter9 polation of the potential field $U$ given boundary constraints $G_{\mathcal{B}}$. The minimization to be solved 

$9 \quad$ setting [12].

\subsection{Additional constraints}

for involve two classical terms that are encountered in the resolution of inverse problems for twodimensional data, for instance in image analysis [26]. The first term is as regularization term computed as a function of the norm of the image gradient. Here, we resort to the total variation criterion, widely exploited for image denoising $[14,26]$. Contrarily to a quadratic criterion, the minimization of the total variation is associated to an anisotropic diffusion, better accounting for geometric image structures. The second term, often referred as the data-driven term, aims at aligning the orientation field of $U$ to orientation constraints $\omega$. A similar term has been used for the reconstruction of dense orientation fields in fingerprint images within a Markovian and quadratic

Minimization (7) is issued from purely geometric constraints. As level-set representations are contrast invariant (i.e., a contrast change does not affect the geometry of the level-sets but only the indexes of the level-sets) [22], additional constraints on $U$ are needed to numerically solve for Eq.(7). For instance, if the boundary set is given by the growth center at time 0 and the final shape at time $T$, solving for Eq.(7) converges to an unstable solution constant everywhere except at the point corresponding to the growth center.

To account for this issue, a theoretical solution would be that the surface between two successive level-lines $\Gamma_{t}(U)$ and $\Gamma_{t+d t}(U)$ is constrained by the instantaneous accretionary deposit at time $t$. Since the actual instantaneous growth rate is generally unknown, we cannot straightforwardly exploit this constraint. However, setting constraints on the surface between successive level-lines is equivalent to setting constraints on first-order statistics $p(U)$ of $U$. In fact, the knowledge of the law of the accretionary deposit comes to set the form of this statistical distribution. Therefore, 
4 subject to $p(U)$ is uniform.

$$
\min _{U \in \mathcal{U}\left(G_{\mathcal{B}}\right)} \int_{p \in \mathcal{R}^{2}}(1-\gamma) \cdot|\nabla U|+\gamma \cdot \alpha \cdot|\langle\nabla U, \omega\rangle|
$$

\section{${ }_{5} 3$ Numerical resolution}

6 To solve for the above constrained minimization, we adopt a two-step iterative approach: the first 7 step comes to project the current solution onto the set of level-representations with uniform first8 order statistics. We detail below the unconstrained minimization. To improve the convergence, a 9 multiresolution framework is adopted.

\subsection{Unconstrained minimization}

To solve for the minimization of $E$ with respect to $U$, Euler-Lagrange equations [1] provide the expression of the gradient of $E$. Given that the $\mathrm{L} 1$ norm is not derivable in zero, it is approximated as $\sqrt{\epsilon^{2}+x^{2}}$. We let the reader refer to [26] for the analysis of the total variation criterion whose gradient is given by:

$$
\frac{\left(\epsilon_{R}^{2}+U_{x}^{2}\right) U_{x x}-2 U_{y} U_{x} U_{x y}^{2}+\left(\epsilon_{R}^{2}+U_{y}^{2}\right) U_{y y}}{\left(\epsilon_{R}^{2}+\nabla U^{2}\right)^{3 / 2}}
$$


Neglecting the variations of the weight $\alpha^{3}$, the second term leads to the following gradient:

$$
\begin{aligned}
\Psi(U) & =\operatorname{div}\left(\frac{\langle\nabla U, \omega\rangle \omega}{\left(\epsilon_{D}^{2}+\langle\nabla U, \omega\rangle^{2}\right)^{1 / 2}}\right) \\
& =\frac{\operatorname{div}(\omega)\langle\nabla U, \omega\rangle}{\left(\epsilon_{D}^{2}+\langle\nabla U, \omega\rangle^{2}\right)^{1 / 2}}+\frac{\epsilon_{D}^{2}\langle\nabla \nabla U \cdot w+\nabla \omega \cdot \nabla U, \omega\rangle}{\left(\epsilon_{D}^{2}+\langle\nabla U, \omega\rangle^{2}\right)^{3 / 2}}
\end{aligned}
$$

1 where div is the divergence operator, $\nabla \nabla U_{p}$ the Hessian matrix of $U$ at point $p$, and $\nabla \omega_{p}$ the

3 gradient matrix $\left(\nabla w_{p, x} ; \nabla w_{p, y}\right)$ with $\omega_{p, x}$ and $\omega_{p, y}$ the horizontal and vertical component of $\omega_{p}$.

The expression of $\Psi(U)$ can be compared to PDEs proposed for anisotropic image smoothing which reduces to $\operatorname{div}(\langle\nabla U, \omega\rangle \omega)$ in the case of the smoothing along a single direction $\omega[28,29]$. The first term is a weighted version of advection term $\langle\nabla U, \omega\rangle$. This advection equation corresponds to the transport of a scalar quantity $U$ preserved by a vector field $\omega$, for instance the transport of temperature by wind and currents in meteorology and oceanography. The weighting factor depends on $\operatorname{div}(\omega)$. If $\omega$ is issued from the actual morphogenesis potential function, $\operatorname{div}(\omega)$ is the curvature of the true (but unknown) successive growth shapes to be reconstructed.

The second term involved in the expression of $\Psi(U)$ is a weighted version of the diffusion operator along the field lines of $\omega, \omega^{T} \nabla \nabla U w+\omega^{T} \nabla \omega \nabla U$, as proposed in [28] for curvature-based regularization of images. As $\sqrt{\epsilon^{2}+x^{2}}$ is used as a numerical approximation of the L1 norm $\|x\|$, $\left|\epsilon_{D}\right|<<1$. Therefore, this second term tends to be negligible.

From these considerations, the steady-state corresponding to evolution equation $d U / d t=\Psi(U)$ reduces to advection equation

$$
\operatorname{div}(\omega)\langle\nabla U, \omega\rangle=0
$$

\footnotetext{
${ }^{3}$ It should be stressed that this confidence map is actually exploited only with orientation fields computed from image gradients.
} 
1 From this steady-state equation, the existence and the uniqueness (up to a contrast change) of the

2 solution of the considered inverse problem can be derived.

3 Proposition 1 Let $\phi$ be a potential function such that $\nabla \phi \neq 0$ and $\operatorname{div}\left(\nabla \phi^{\perp} /|\nabla \phi|\right) \neq 0$. Let $\omega$ be

4 the orientation field tangent to the level-lines of $\Phi$.

${ }_{5} \quad$ Let $U$ be a potential function such that $\nabla U \neq 0 . U$ is a solution of Eq.11 if and only if it exists

$6 \quad$ a strictly monotonic continuous and derivable function $g$ such that $U=g(\Phi)$.

7 Proof. As $\omega=\nabla \phi^{\perp} /\left|\nabla \Phi^{\perp}\right|, \operatorname{div}(\omega) \neq 0$. Hence, Eq.11 is equivalent to $\langle\nabla U, \omega\rangle=0$. As the 8 level-lines of a field $U$ can be defined as the lines tangent to $\nabla U^{\perp}$, the latter equation imposes 9 level-lines of $U$ and $\Phi$ to be aligned. It then exists a continuous and derivable function $g$ such that 10 $U=g(\Phi)$. Given that $\nabla U \neq 0$ and $\nabla \phi \neq 0, g$ is strictly monotonic.

11 Reciprocally, potential function $U=g(\Phi)$, with $g$ a strictly monotonic continuous and derivable function, is a solution of Eq.11 $\square$.

This proposition guarantees that, if direction field $\omega$ is the direction field of an unknown potential function, the solutions of Eq.11 are transformed versions of this unknown potential function with respect to a contrast change. Note also that this is only valid for a domain on which $\operatorname{div}\left(\nabla \phi^{\perp} /|\nabla \phi|\right) \neq 0$. Assuming that only isolated points do not fulfill this condition, this results can be extended to the whole domain by continuity. Condition $\operatorname{div}\left(\nabla \phi^{\perp} /|\nabla \phi|\right) \neq 0$ indeed resorts to considering that the curvature of the level-lines is not null. Formally, such an assumption seems reasonable for the studied biological phenomena: they rather involve round or elliptic growth shapes and the formation of growth shapes involving perfect straight lines seems unlikely. From a numerical point of view, numerical unstabilities have however been observed in practice when using directly the gradient-descent issued from Euler-Lagrange equations. These numerical unstabilities may be due to weighting factor $\operatorname{div}(\omega)$ since growth shapes far from the growth center tend to 
become locally closer to straight lines. Consequently, to ensure a better numerical resolution, we prefer exploiting an explicit Gauss-Seidel scheme. Given that Eq.11 does not lead to a linear system, the Gauss-Seidel scheme is derived from the discrete version of the considered variational criterion. Considering a finite difference approximations of $|\nabla U|$ similar to Markovian setting [17, 23], we resort to:

$$
(1-\gamma) \sum_{(p, q) \in \mathcal{N}} \rho_{\epsilon_{R}}\left(U_{p}-U_{q}\right)+\gamma \sum_{p \in \mathcal{R}^{2}} \alpha_{p} \cdot \rho_{\epsilon_{D}}\left(\left\langle\nabla U_{p}, \omega_{p}\right\rangle\right)
$$

where $\mathcal{N}$ is the four-neighborhood system and (i.e., the neighborhood of a point is given its four western, northern, eastern and southern neighbors in the image). The minimization of the discrete energy involving robust functions is carried out using an iterated reweighted least square (IRLS) scheme, which iterates two steps: the computation of robust weights

$$
\beta_{R}(p, q)=\phi_{R}\left(U_{p}-U_{q}\right)
$$

and

$$
\beta_{D}(p)=\phi_{D}\left(\left\langle\nabla U_{p}, \omega_{p}\right\rangle\right)
$$

where function $\phi$ is the influence function associated with robust estimator $\rho$ computed as $\phi(x)=$ $\rho^{\prime}(x) / x$ (these robust weights are low for high residual error, i.e. outliers, and high for low residuals); and solving for the weighted least square minimization

$$
\min _{U \in \mathcal{U}\left(G_{\mathcal{B}}\right)}(1-\gamma) \sum_{(p, q) \in \mathcal{N}} \beta_{R}(p, q) \cdot\left|U_{p}-U_{q}\right|^{2}+\gamma \sum_{p \in \mathcal{R}^{2}} \alpha_{p} \cdot \beta_{D}(p) \cdot\left\langle\nabla U_{p}, \omega_{p}\right\rangle^{2}
$$

1 given robust weight maps $\beta_{R}$ and $\beta_{D}$. This least square estimation is indeed solved for using a

2 Gauss-Seidel scheme. $U_{p}$ is iteratively updated by explicitly solving for the quadratic minimization 3 (15) under the assumption that $U$ is constant except at point $p$. The associated computations 4 based on a centered numerical approximation of $\nabla U_{p}$ are detailed in Annex. 


\section{$1 \quad 3.2 \quad$ Reprojection onto the constrained subspace}

2 The projection of the current solution $U$ onto the set of level-set representation with uniform first3 order statistics can be viewed as an histogram equalization. As histogram equalization can be 4 implemented as a PDE scheme [6], constrained minimization (8) could be solved by coupling the 5 PDE issued from the gradient of $E$ to the one proposed in [6]. However, as stressed above, such 6 a PDE-based scheme is numerically unstable and a discrete Gauss-Seidel minimization scheme is 7 preferred. The histogram equalization is applied as a second step after the Gauss-Seidel loop. Given

$8 \tilde{U}$, the distribution of the potential values $p(\tilde{U}$ is computed as well as their cumulative distribution ${ }_{9} F(\tilde{U})$. Aligning $F(\tilde{U})$ to line $y=x$ provides us with a contrast change approximately leading

\subsection{Multiresolution minimization}

Minimization (7) is obviously not convex. To ensure a better robustness to the initialization, a multiresolution scheme is used. Given a Gaussian pyramid of the orientation field $\omega[2]$, the multiresolution approach comes to solve for the estimation of the potential function $U$ at successive resolutions, from the coarsest resolution to the finest one. The final estimate at a given resolution serves as the initialization at the next resolution. In practice, Gaussian pyramids with four levels were exploited.

\subsection{Initialization}

The computation of a relevant initialization to the proposed gradient-based multiresolution scheme is important to ensure a fast and relevant convergence. The initialization is delivered by the 

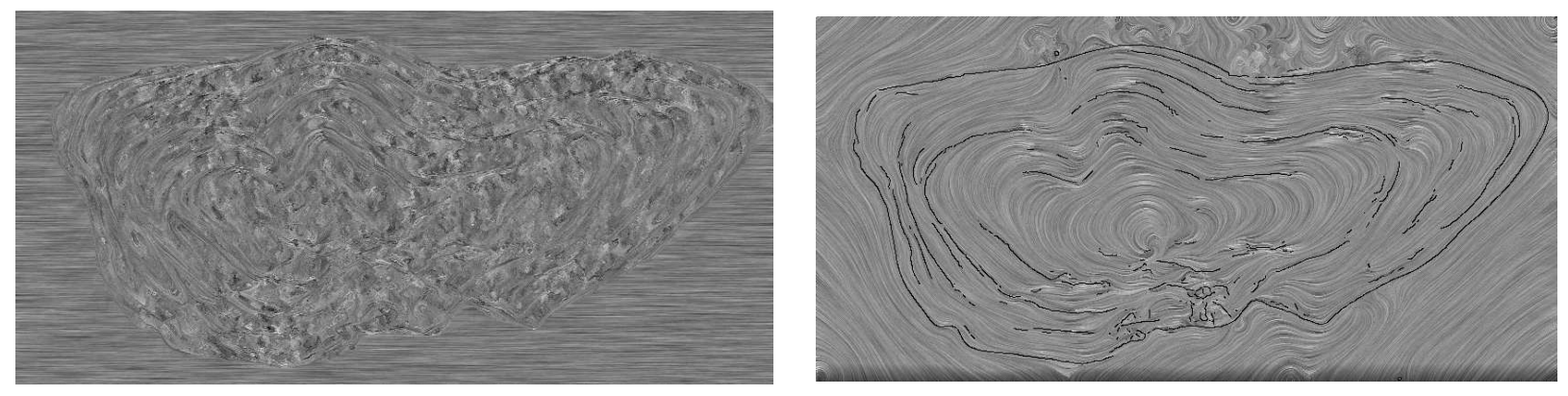

Figure 4: Examples of extracted growth orientation fields from local image orientations for the image of a pollock (Pollachius pollachius) otolith depicted in Fig.1: visualization of the orientation field issued from the image gradient (left), visualization of the orientation field computed as the AMLE (Absolutely Minimizing Lipschitz Extension) of automatically selected gradient measures depicted by black lines (right). Orientation fields are visualized via their field lines using LIC) [3].

1 scalar AMLE (Absolutely Minimizing Lipschitz Extension) of the boundary conditions to the whole 2 domain [7]. Since the AMLE resorts to the minimization of the $L_{\infty}$ norm, the AMLE can be viewed 3 as the less smooth interpolation operator among all smooth interpolation operator. The AMLE is 4 implemented as gradient descent $\partial U / d t=U_{\nabla \nabla}$ where $U_{\nabla \nabla}$ is the second-order derivative in the 5 direction of the gradient of $U$. It is shown to converge to the unique AMLE. The key properties 6 of the AMLE is that it is an artifact-free and oscillation-free interpolant actually capable of taking 7 into account isolated boundary points as well as curves.

\section{${ }_{8} 4$ Results}

\section{$9 \quad 4.1 \quad$ Extraction of the orientation fields}

10 The proposed approach initially relies on the extraction of orientation field $\omega$. As detailed previ-

11 ously, orientation field $\omega=\nabla I /|\nabla I|$ computed from the image gradient $\nabla I$ is first investigated. The second solution comes to computing $\omega$ as the AMLE of the orientations of the gradient measures 
selected by a thresholded Canny-Deriche edge filter $[9,13]$. Fig. 4 reports the orientation fields issued from these two schemes for the image depicted in Fig.1. These orientation fields are visualized via their field lines, using Line Integral Convolution (LIC) [3]. As expected, orientation field $\omega=\nabla I /|\nabla I|$ is not globally coherent and only orientation information along image contours may be meaningful. On the contrary, the AMLE orientation field is much smoother. It conveys relevant geometric cues, which are expected to better constrain the reconstruction of the 2D morphogenesis.

\subsection{Examples of reconstruction of the series of growth shapes}

To illustrate the different steps of our approach, we first display the reconstruction of the series of growth shape for the pollock otolith image previously depicted in Fig.1. The results of the reconstructions of the morphogenesis associated with the orientation fields issued from the image gradient and the AMLE scheme are compared in Fig. 5. Besides, reconstructions issued for two different configurations of boundary constraints $G_{\mathcal{B}}$ : the first example only involves the position of the growth center, whereas the growth center as well as the first translucent ring is given in the second example. Parameters $\gamma, \epsilon_{R}$ and $\epsilon_{D}$ are respectively set to $0.6,0.1$ and 0.1 for the first case and to $0.9,0.1$ and 0.1 for the latter. Equally sampled level lines $\left\{\Gamma_{t}(U)\right\}$ superimposed to the otolith image are reported. Due to the weaker coherence of the gradient-based orientation field, the minimization of the variational criterion does not lead to very regular level lines in that case. If only the growth center is provided, such orientation information is not sufficient to reconstruct relevant growth shapes, especially for the first two rings. The additional boundary constraint given as the first bright ring permits to greatly improve the reconstruction of the series of growth shape. The results issued from the AMLE orientation field clearly depict more regular shapes which fit to the internal otolith structures. In addition, the two boundary constraints lead to very similar 
results: few differences can be observed and mainly occur in the ventral lobe (left part) of the first translucent ring. Concerning computational time, the proposed scheme is implemented as a C code under linux and runs in about half a minute for a 500x1000 image.

To further illustrate the role of the two energy terms involved in the proposed variational formulation, results are reported for the two configurations processed in Fig.6 and Fig.7 setting $\gamma$ to 0 and 1 . If only the regularization term is exploited, the reconstruction of the shapes only relies on the geometric prior set in terms of shape regularity and can be viewed as a shape matching example $[6,30]$. Such prior does not however permit to reconstruct relevant shape deformations if only the growth center and the otolith outline is provided. The quality of the reconstruction clearly improves if additional boundary constraints are added. While results reported using the AMLE orientation field appear very similar for $\gamma=1$ (Fig.7) and $\gamma=0.9$ (Fig.5). It is however preferable to impose some minimum regularity (i.e., $\gamma<1$ ) in order to deal with potential local incoherences of the AMLE orientation field. This is indeed further illustrated when the gradient orientation-field are used, since the minimization of the variational criterion with $\gamma=1$ does not lead to a meaningful configuration even if the first translucent ring is provided (Fig.7). Practically, a trade-off has to be achieved between regularity and conformity to the orientation field.

With a view to further investigating this aspect, experiments are carried out for synthetic data. The synthetic image is created as follows. For the otolith image depicted in Fig.1, the boundary constraints defined by the set of all translucent rings were exploited to extract a sequence of otolith shapes using only the regularization term (i.e., $\gamma=0$ ). The values of potential function $U$ are then converted to intensity using a modulated sinusoidal mapping to generate an image depicting a sequence of dark and bright rings. This image is corrupted by a centered Gaussian noise with a standard deviation of 10 . This test image is associated with a reference potential function $U_{G T}$ 
which can be used as a reference for performing a quantitative evaluation. Such a quantitative evaluation is reported in Fig.8. Both the performances of the variational setting combined to gradient-based and AMLE orientation fields were evaluated as a function of the weighting factor $\gamma$.

These results further stress that the AMLE scheme is more robust and more stable. As confirmed empirically for real images, $\gamma$ values between $[0.8,1[$ correspond to the best trade-off between regularity and conformity to orientation data.

To further illustrate the flexibility of the proposed scheme, an example of reconstruction of the growth shapes is carried out using additional constraints given as annotated segments of an internal ring (Fig. 9). This example demonstrates the capability of the proposed variational scheme to take such constraints into account in order to improve the reconstruction of the shapes compared to the results reported above.

Experiments for three other fish species, namely plaice (Pleuronectes platessa), cod (Gadhus morua) and hake (Merluiccius merluccius), as well as the section of a tree trunk, are presented in Fig.10) and Fig.11). Obviously, better results are recovered for the plaice otolith and the section of a tree trunk, which involves the clearest structure. The results reported for the whiting (Gadhus morua) and cod (Merlangius merlangus) otoliths demonstrate that we are also capable of approximately recovering the complex and non-uniform evolution of such shapes from lower-contrasted images.

\section{Discussion}

We have proposed a scheme aimed at reconstructing from an image the evolution of the shape of biological structures involving accretionary growth process. Its key feature is a level-set representation, which intrinsically accounts for the major characteristics of the accretionary growth process. 
From purely geometric criteria on shape regularity and on local orientation coherence with respect to the observed image, a variational formulation is derived. The associated minimization is efficiently solved for within a discrete robust multiresolution framework. Reported results on synthetic and real data provides a validation of the proposed approach. In particular, it demonstrates that AMLE orientation fields should be preferred to the orientation of image gradient since it conveys more relevant geometric information on image structures than image gradients.

Future work will investigate several refinements and extensions of the present work. Whereas uniformity constraints set to the distribution of potential function $U$ are exploited to solve for minimization (7), other constraints more closely related to growth dynamics (for instance, a mean growth model) might also be exploited. New regularization term $E_{R}(U)$ may also be developed to encode a priori knowledge on shape statistics, for instance using an exemplar-based setting [11]. 3D morphogenesis reconstruction may also be investigated as an extension of this work from the joint analysis of several observation planes. This contribution also provides the basis for the development of improved pre-processing (for instance, orientation-based image filtering) and information extraction (growth axis, growth rings) tools [10] for the considered biological images. In the field of marine ecology, new issues can also be investigated in terms of understanding and modeling of biocalcification and accretionary growth process.

\section{References}

[1] G. Aubert and P. Kornprobst. Mathematical problems in image processing. Springer-Verlag, New York, USA, 2002.

[2] P.J. Burt. The pyramid as a structure for efficient computation. Springer-Verlag, 1984. In 
A.Rosenfeld, éditeur, pages 6-35.

[3] B. Cabral and L.C. Leedom. Imaging vector field using line integral convolution. Computer Graphics Proceedings, pages 263-270, 1993.

[4] S. Campana and J. Casselman. Stock discrimination using otolith shape analysis. Can. Jal of Fish. Res. and Aqu. Sc., 50:1062-1083, 1993.

[5] F. Cao and R. Fablet. Automatic morphological detection of otolith nucleus. Pattern Recognition Letters, 2006.

[6] V. Caselles, , and G. Sapiro. Histogram modification via partial differential equations. J. Diff. Equations, 135:238-268, 1997.

[7] V. Caselles, J.-M. Morel, and C. Sbert. An axiomatic approach to image interpolation. IEEE Trans. on Image Processing, 7:376-386, 1998.

[8] L. Chauvaud. The shell of the Great Scallop Pecten maximus as a tool for high frequency paleoenvironmental reconstruction. Geochemistry, Geophysics, Geosystems 6, 2004. In press.

[9] A. Chessel, F. Cao, and R. Fablet. Interpolation of orientation: an axiomatic approach. In Proc. of European Conference on Computer Vision, ECCV06, Graz, May 2006.

[10] A. Chessel, R. Fablet, and F. Caoand C. Kervrann. Orientation interpolation and applications. In Proc. of 13th Int. Conf. on Image Processing, ICIP'2006, Atlanta, October 2006.

[11] D. Cremers and C. Schnörr. Statistical shape knowledge in variational motion segmentation. Image and Vision Computing, 21(1):77-86, 2003. 
$1 \quad[12]$ S. Dass. Markov random field models for directional field and singularity extraction in fingerprint images. IEEE Trans. on Image Processing, 13(10):1358-1367, 2004.

[13] R. Deriche. Using canny's criteria to derive a recursively implemented optimal edge detector. International Journal of Computer Vision, 1(2):167-187, 1987.

[14] S. Durand and J. Froment. Reconstruction of wavelet coefficients using total variation minimization. SIAM Jal Scientific Computing, 24(5):1754-1767, 2003.

[15] R. Fablet. Semi-local extraction of ring structures in images of biological hard tissues: application to the bayesian interpretation of fish otoliths for age and growth estimation. Canadian Journal on Fisheries and Aquatic Sciences, 43(6):1414-1428, 2006.

[16] R. Fablet and F. Daverat andH. de Pontual. Unsupervised bayesian reconstruction of individual life histories chronologies from otolith signatures: case study of Sr:Ca transects of eel (anguilla anguilla) otoliths. Can. Jal of Fish. Res. and Aqu. Sc., 2006. Submitted.

[17] S. Geman and D. Geman. Stochastic relaxation, Gibbs distribution and the Bayesian restoration of images. IEEE Trans. on Pattern Analysis and Machine Intelligence, 6(6):721-741, 1984.

[18] R. Gonzalez and R. Woods. Digital image procesing. Addison-Wesley Publishing Company, 1992.

[19] A. Guillaud, A. Benzinou, H. Troadec, V. Rodin, and J. Le Bihan. Autonomous agents for edge detection and continuity perception on otolith images. Im. and Vis. Comp., 20(13-14):955-968, 2002.

[20] P. Huber. Robust Statistics. John Wiley \& Sons, 1981. 
1 [21] F. Lin and X. Yang. Geometric Measure Theory: An Introduction. International Press, 2002.

2 [22] P. Monasse and F. Guichard. Fast computation of a contrast invariant representation. IEEE Trans. on Image Processing, 9(5):860-872, 2000.

[23] E. Mémin and P. Pérez. A multigrid approach for hierarchical motion estimation. In Proc. of 6th IEEE Int. Conf. on Computer Vision, ICCV'98, pages 933-938, Bombay, Jan. 1998.

[24] J.M. Odobez and P. Bouthemy. Separation of moving regions from background in an image sequence acquired with a mobile camera. In Video Data Compression for Multimedia Computing, chapter 8, pages 295-311. H. H. Li, S. Sun, and H. Derin, eds, Kluwer, 1997.

[25] J. Panfili, H. De Pontual, H. Troadec, and P.J. Wright. Manual of Fish Sclerochronology. Editions Ifremer, 2003.

[26] L. Rudin, S. Osher, and E. Fatemi. Nonlinear total variation based noise removal algorithms. Physica D, 60(1-4):259-268, 1992.

[27] D. W. Thompson. On growth and form. Cambridge University Press, 1917.

[28] D. Tschumperlé. Fast anisotropic smoothing of multi-valued images using curvature-preserving PDE's. International Journal of Computer Vision, 0(0):xx-xx, 2006.

[29] J. Weickert. Anisotropic diffusion in image processing. Teubner-Verlag, Stuttgart, 1998.

[30] L. Younès. Optimal matching between shapes via elastic deformations. Image and Vision Computing, 17:381-389, 1999. 


\section{Appendix: potential update}

The local update of potential $U$ at point $p$ to solve for Eq.15 relies on a centered gradient approximation and is computed as:

$$
U_{p}=\frac{(1-\gamma) \sum_{q \in \mathcal{N}_{p}} \beta_{R}(p, q) \cdot U_{q}+\gamma \sum_{q \in \mathcal{N}_{p}^{H}} \beta_{D}(q) \omega_{q, x}\left\langle A_{p, q}, \omega_{q}\right\rangle+\gamma \sum_{q \in \mathcal{N}_{p}^{V}} \beta_{D}(q) \omega_{q, y}\left\langle B_{p, q}, \omega_{q}\right\rangle}{(1-\gamma) \sum_{q \in \mathcal{N}_{p}} \beta_{R}(p, q)+\gamma \sum_{q \in \mathcal{N}_{p}^{H}} \beta_{D}(q) \omega_{q, x}^{2}+\gamma \sum_{q \in \mathcal{N}_{p}^{V}} \beta_{D}(q) \omega_{q, y}^{2}}
$$

2 where $\mathcal{N}_{p}$ comprises the four neighbors of $p, \mathcal{N}_{p}^{H}$ its two horizontal neighbors and $\mathcal{N}_{p}^{H}$ its two 3 vertical ones. $\omega_{q, x}$ and $\omega_{q, y}$ are respectively the horizontal and vertical component of vector $\omega_{q}$.

4 Vector $A_{p, q}$ for point $p=(i, j)$ and its horizontal neighbor $q=(i, j+k), k=\mp 1$, is defined as $5\left(U_{i, j+2 k},(-1)^{\frac{k+1}{2}}\left(U_{i+1, j+k}-U_{i-1, j+k}\right)\right)^{t}$. Similarly, vector $B_{p, q}$ for point $p=(i, j)$ and its vertical 6 neighbor $q=(i+k, j), k=\mp 1$, is defined as $\left(U_{i, j+2 k},(-1)^{\frac{k+1}{2}}\left(U_{i+1, j+k}-U_{i-1, j+k}\right)\right)^{t}$. 

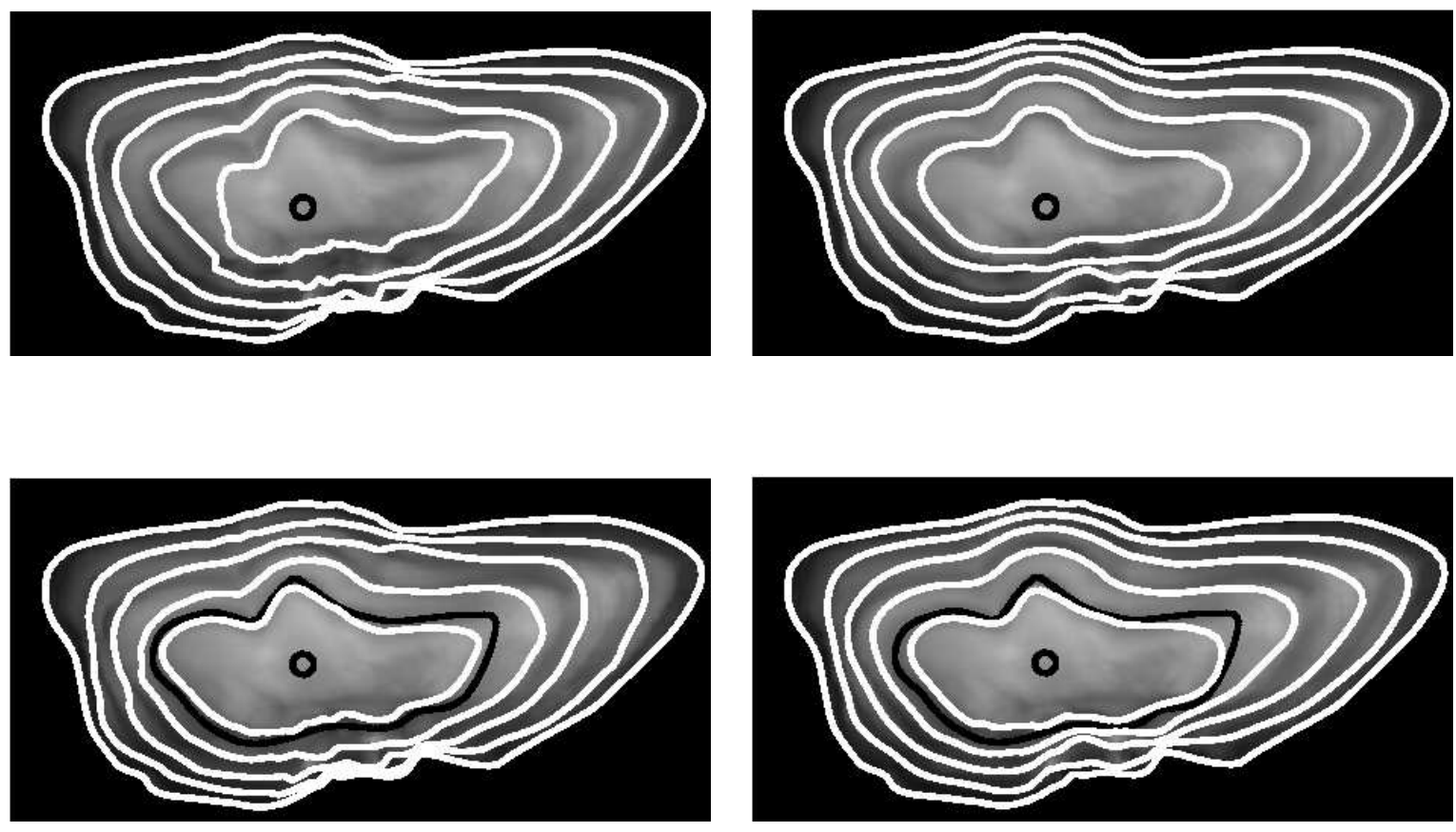

Figure 5: Reconstruction of the series of growth shapes for the pollock (Pollachius pollachius) otolith image depicted above: first row, reconstruction given the otolith center using the gradient-based (left) and AMLE (right) orientation fields; second row, reconstruction given the otolith center and the first opaque ring using the gradient-based (left) and AMLE (right) orientation field. Parameter $\epsilon$ is set to 0.1. The constraints (i.e., the otolith center and the first opaque ring) are superimposed to the otolith image as black curves, and the equally sampled level sets of the reconstructed potential function $U$ as white curves. 

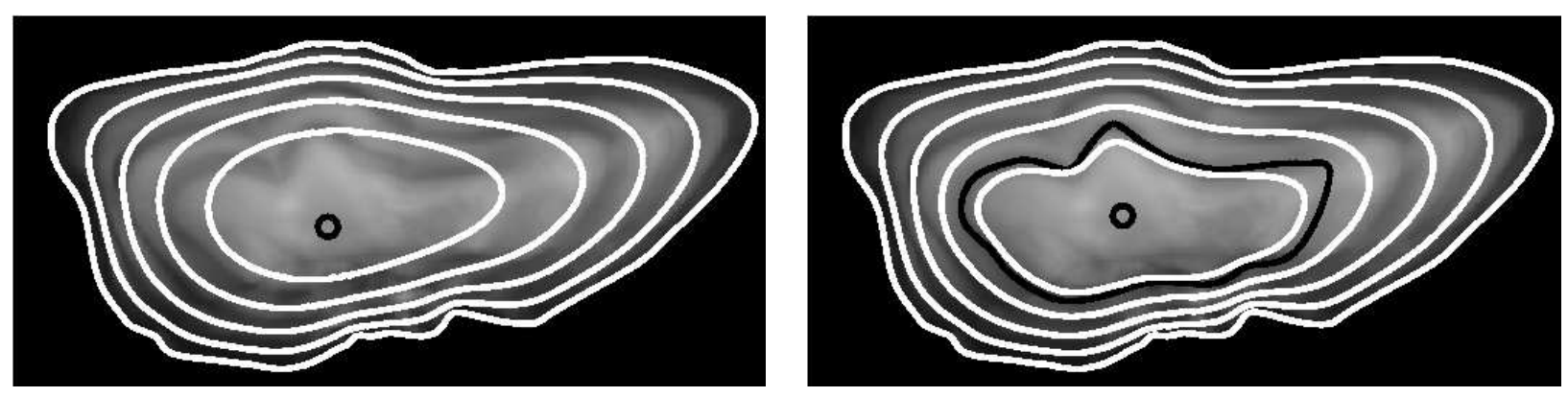

Figure 6: Reconstruction of the series of growth shapes for the pollock (Pollachius pollachius) otolith image depicted above setting $\gamma$ to 0 (i.e., using only the regularization term): left, given the otolith center; right, given the otolith center and the first opaque ring. In both cases, the constraints (i.e., the otolith center and the first opaque ring) are superimposed to the otolith image as black curves, and the equally sampled level sets of the reconstructed potential function $U$ as white curves. 

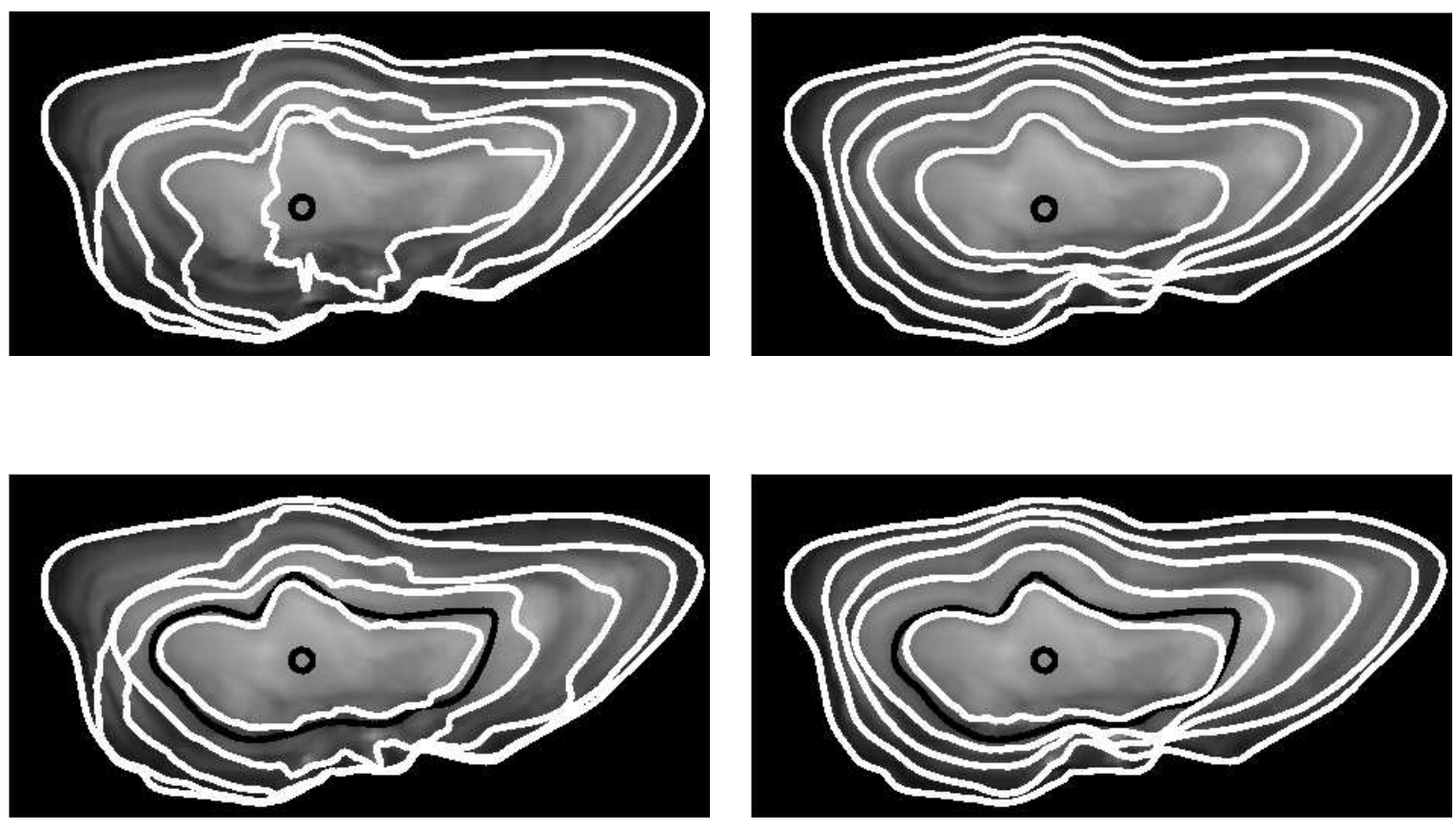

Figure 7: Reconstruction of the series of growth shapes for the pollock (Pollachius pollachius) otolith image depicted above setting $\gamma$ to 1 (i.e., using only the data-driven term): first row, results are obtained knowing the position of the growth center using the gradient-based (left) and AMLE (right) orientation fields; second row, reconstruction knowing the otolith center and the first opaque ring using the gradient-based (left) and AMLE (right) orientation field. Parameter $\epsilon$ is set to 0.1 and weighting factor. The constraints (i.e., the otolith center and the first opaque ring) are superimposed to the otolith image as black curves, and the equally sampled level sets of the reconstructed potential function $U$ as white curves. 

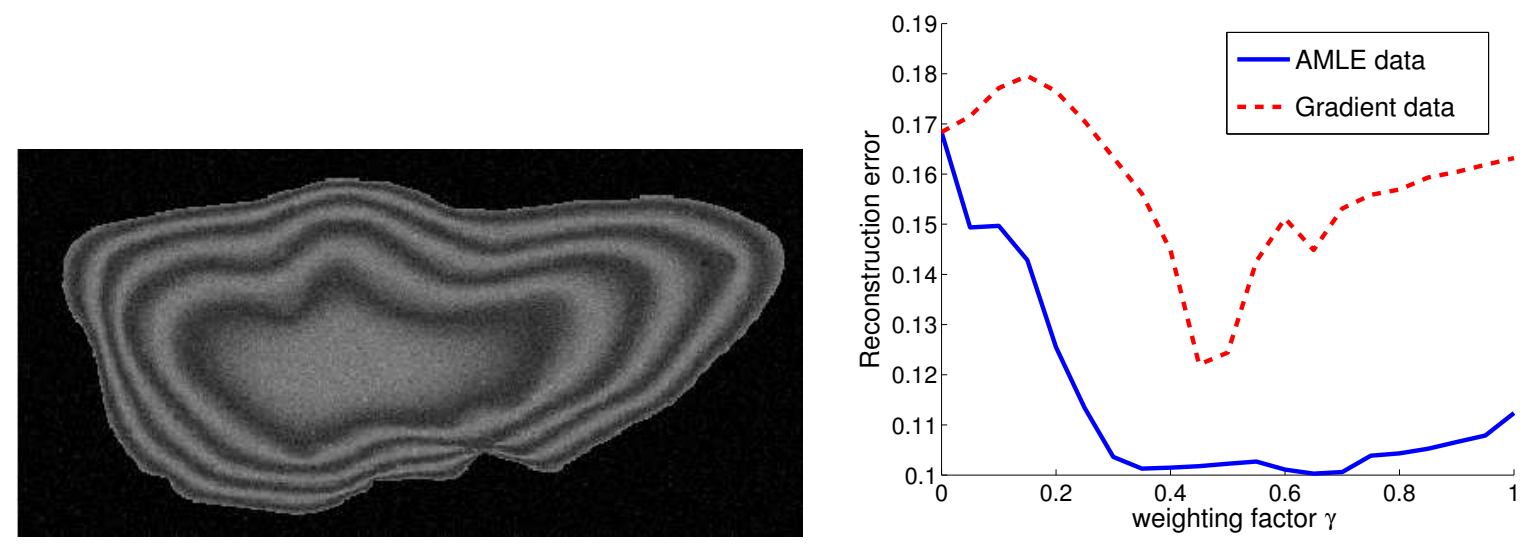

Figure 8: Influence of the weighting factor $\gamma$ on the reconstruction error for a synthetic otolith image: left, noisy synthetic otolith image; right, reconstruction error using gradient-based and AMLE orientation field as a function of the weighting factor $\gamma$.
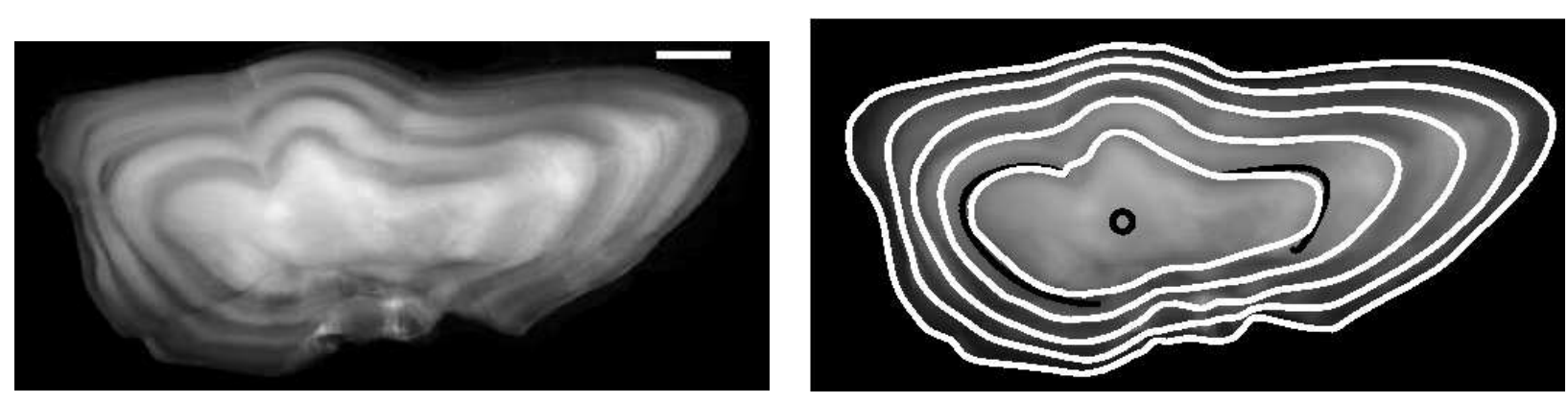

Figure 9: Reconstruction of the series of growth shapes for the pollock (Pollachius pollachius) otolith processed for boundary constraints given by the position of the growth center and two segments of the first opaque ring: original image (left), series of shape superposed to the otolith image (right column). These experiments have been carried out using the AMLE orientation field to compute the data-driven term. Boundary constraints are depicted as black curves in the images. 

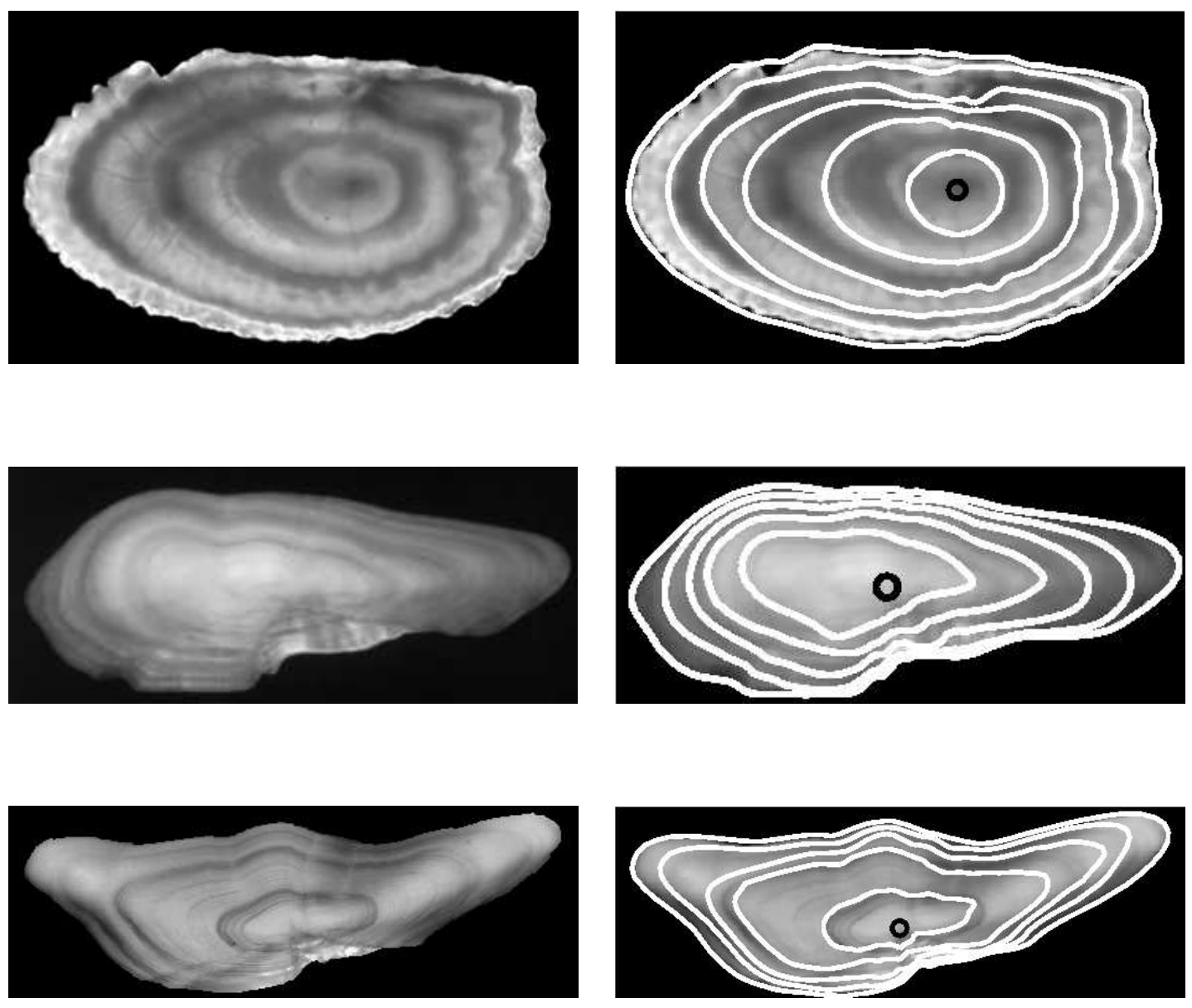

Figure 10: Reconstruction of the series of growth shapes for three examples of fish otoliths: $a$ plaice (Pleuronectes platessa) otolith (first row), a cod (Gadhus morua) otolith (second row), and a whiting (Merlangius merlangus) otolith (third row. For each row, the otolith image and the series of shape superposed to the otolith image are reported. The same parameter setting as in the previous experiments is used. 

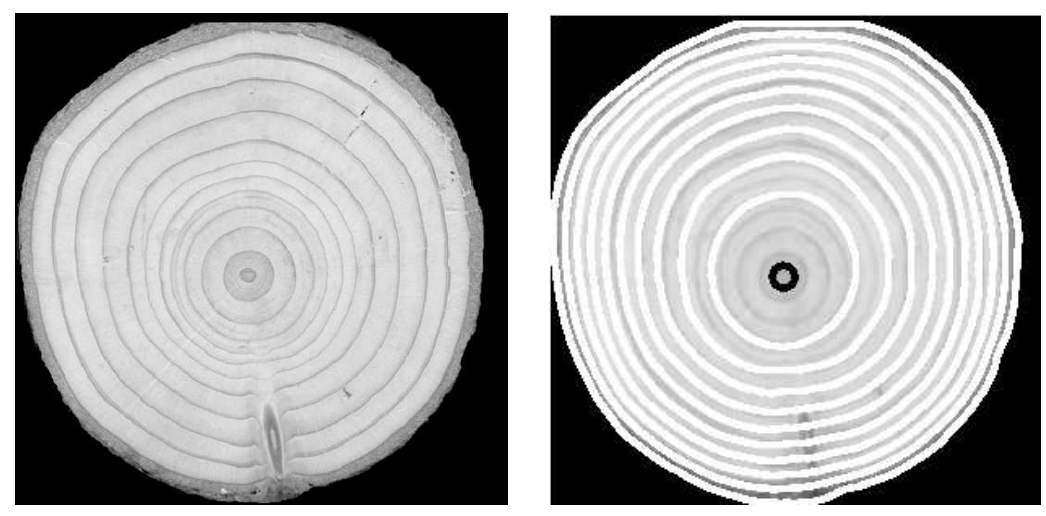

Figure 11: Reconstruction of the series of growth shapes of the section of a tree trunk: original image (left), reconstructed potential function $U$ (left column), series of shape superposed to the image of the section of the tree trunk (right column). These experiments have been carried out using the AMLE orientation field to compute the data-driven term. The growth center is depicted as a black circle. 